RAD Conference Proceedings, vol. 2, pp. 59-63, 2017

www.rad-proceedings.org

novororitty

\title{
MONTE CARLO SIMULATIONS OF THIN FILMS LOADED WITH OSL DETECTORS
}

\author{
N. Mirzajani1 ${ }^{*}$, S.O. Souza' ${ }^{1}$ F. d'Errico' ${ }^{1,2,3}$ \\ ${ }^{1}$ Departamento de Física, Universidade Federal de Sergipe, São Cristóvão, SE, Brazil \\ ${ }^{2}$ Scuola di Ingegneria, Università di Pisa, Pisa, Italy \\ 3Yale University, School of Medicine, New Haven, CT, USA
}

\begin{abstract}
The transport and interactions of gamma-rays in a thin film loaded with optically stimulated luminescence (OSL) nanoparticles (NPs) were studied by Monte Carlo (MC) simulations with the Particle and Heavy Ion Transport code System (PHITS). In the MC input file, the geometry of the thin film was treated as a virtual space using a cubic voxel structure with a lattice of nanoparticles (NPs) of OSL $\mathrm{CaF}_{2}:$ Ce. The particles were monodispersed, ranging in size from 50 to $600 \mathrm{~nm}$. The polyvinyl chloride (PVC) film matrix was treated as an array simulating a small sample with an area of $13.8 \mu \mathrm{m}$ by $13.8 \mu \mathrm{m}$ and a thickness of $10.2 \mu \mathrm{m}$. For the irradiation simulations, we considered a collimated beam of cesium-137 gamma-rays of $662 \mathrm{keV}$ impinging perpendicularly on the piece of thin film (detector). The film was centered on the front face of $30 \mathrm{~cm} \times 30 \mathrm{~cm} \times 15 \mathrm{~cm}$ ISO water slab phantom. In the MC simulations, we followed the radiation tracks and calculated the energy deposition from the tracks of electrons produced by the interaction histories of the photons crossing the thin film. The energy deposition in the OSL film is initially fairly constant with grain size and then increases as the $\mathrm{CaF}_{2}$ :Ce grains get larger to the point of filling $5 \mathrm{O} \%$ of the voxel volume. For grain sizes up to almost $400 \mathrm{~nm}$, the presence of the grains has minimal impact, i.e., the dose is mainly deposited by secondary electrons generated within the polymer. This allows for the design of tissueequivalent dosimeters even with embedded OSL materials, such as $\mathrm{CaF}_{2}$, that exhibit a higher Z-value than tissue.
\end{abstract}

Key words: Dosimetric film, Optically Stimulated Luminescence, Monte Carlo simulation

DOI: $10.21175 /$ RadProc.2017.13

\section{INTRODUCTION}

In recent years, the development of nanometer scale grains of OSL materials has been reported [1-2] and has attracted interest for a variety of radiation dosimetry applications. Among these is the development of detectors for $2 \mathrm{D}$ or $3 \mathrm{D}$ dose mapping.

Technologies based on OSL films have been developed in several laboratories worldwide. First, films containing carbon-doped aluminum oxide $\left(\mathrm{Al}_{2} \mathrm{O}_{3}: \mathrm{C}\right)$ were developed in the $1980 \mathrm{~s}$ at Pacific Northwest National Laboratory as a commercial OSL dosimetry system for radiation protection [3]. Since 2000, $\mathrm{Al}_{2} \mathrm{O}_{3}: \mathrm{C}$ powder mixed with a polyester binder and coated onto a roll of polystyrene film has been used by the company LANDAUER [4]. In 2006, the performance of a film based on $\mathrm{Al}_{2} \mathrm{O}_{3}: \mathrm{C}$ was studied at radiation oncology and nuclear accident dose levels [5, 6]. In 2011, Nakhaei et al. produced $\mathrm{PVA} / \mathrm{CaF}_{2}$ nanoparticle films with different amounts of $\mathrm{CaF}_{2}$ nanoparticles [7].

In 2011, Luszik-Bhadra described the neutroninduced signals in $\mathrm{Al}_{2} \mathrm{O}_{3}: \mathrm{C}$ films with $10 \mu \mathrm{m}$ to $100 \mu \mathrm{m}$ spherical grains by track-structure calculations using PHITS [8]. Neutron irradiations were found to deposit energy more effectively in $\mathrm{Al}_{2} \mathrm{O}_{3}: \mathrm{C}$ grains with a $10 \mu \mathrm{m}$ diameter compared to grains of $100 \mu \mathrm{m}$ by a factor of three. In the case of photon irradiations, there was no effect changing the diameter of the spherical grains of $\mathrm{Al}_{2} \mathrm{O}_{3}: \mathrm{C}$.

None of the dosimeters mentioned above allows the measurement of the entrance dose closely adhering to the body surface of a patient undergoing a radiological procedure. Conversely, measuring entrance exposures to diagnostic or therapeutic radiology patients would be valuable to infer dose distributions within their body, and to assess and minimize exposures to the skin.

Recently, Souza et al. reported the proof of concept of a novel technical textile for use in dosimetry [9]. They demonstrated that fabrics made of impermeable and flexible fibers or polymeric films loaded with OSL emitting micro- or nano-particles would be a completely new dosimetry method for 2D measurements with garments adhering closely to the body. These could be placed over radiotherapy patients, for example, to measure entrance doses in small field dosimetry, irregular surface dosimetry, and possibly allow postal audits of modulated treatment delivery. This recent approach is based on dispersing micro and nanoparticles inside a polyvinyl chloride (PVC) polymer matrix film using a manufacturing

*nmirzajani12@gmail.com 
methodology similar to that used to disperse nanoparticles in fibers for drug delivery [10].

In this work, we analyzed these dosimeter films with dispersed luminescent micro or nanoparticles. An accurate three-dimensional Monte Carlo (MC) simulation was deemed necessary to optimize the design of our new OSL films. The multi-purpose Monte Carlo Particle and Heavy Ion Transport code System (PHITS) was chosen to assess the transport and collision of ionizing radiation through our new dosimeters [11-13]. The code simulates the transport of nearly all particles, including neutrons, protons, pions, heavy ions (nuclei), muons, electrons, and photons, with wide energy ranges from $\mu \mathrm{eV}$ up to $200 \mathrm{GeV}$ using several nuclear reaction models and nuclear data libraries. Among the relevant features of this code are its ability to simulate the tracks of secondary particles, explicitly handling ionizations and excitations eventby-event.

In this paper, we investigated different diameters of OSL particles of CaF2:Ce embedded in the PVC thin film for assessing the effect of grain size on gamma-ray sensitivity. Our main goal was to optimize the design of the new kind of dosimetric film loaded with OSL micro and nanoparticles. We analyzed the response to Cs-137 photons of 662-keV energy for the assessment of the microfilm since Cs-137 is commonly used as calibration source in laboratories. We used the MC PHITS code to simulate the geometry of the particle dispersion and to investigate the track structure, deposited energy distributions, and calculated the dose due to the transport of secondary particles produced by photon interactions through the thin films.

\section{METHODS}

PHITS version 2.64 was used in this study $[14,15]$. In our analysis of the complex film dosimeter structure, the simulation geometry we used was a virtual space based on a voxel phantom model. We assumed a lattice consisting of a $23 \times 23 \times 17$ matrix of cubic voxels, i.e., 8993 units of $600 \mathrm{~nm} \times 600 \mathrm{~nm} \times 600 \mathrm{~nm}$. Each voxel contained a $\mathrm{CaF}_{2}$ :Ce sphere inside the PVC matrix. We tried to find the size of the particles that produce the best OSL signal related to the absorbed dose. Thus, we investigated films with different diameters of OSL micro and nanoparticles of $\mathrm{CaF}_{2}$ : $\mathrm{Ce}$.

The particles were embedded in polyvinyl chloride (PVC) thin films. The diameter of the $\mathrm{CaF}_{2}$ :Ce spheres was uniform throughout the film. For each new simulation, the grain diameter was changed in the range from $50 \mathrm{~nm}$ to $600 \mathrm{~nm}$. The voxels were arranged to form a $3 \mathrm{D}$ array simulating a piece of film with a surface of $13.8 \mu \mathrm{m} \times 13.8 \mu \mathrm{m}$ and a thickness of $10.2 \mu \mathrm{m}$, loaded with $\mathrm{CaF}_{2}$ :Ce OSL grains. The thin film (detector) was placed at the center of the front face of an ISO water slab phantom $(30 \mathrm{~cm} \mathrm{x} 30 \mathrm{~cm} \mathrm{x} 15 \mathrm{~cm})$ with walls made of PMMA (front wall $2.5 \mathrm{~mm}$ thick and other walls $10 \mathrm{~mm}$ thick) and filled with water. We used appropriate tallying to score the parameters of interest. Namely, we ran the PHITS program using the T-Gshow tally for checking the geometry in a 2-dimensional view. The 2D geometry (XZ plane) of the ISO water slab phantom with the film detectors on the front is shown in Figure 1.

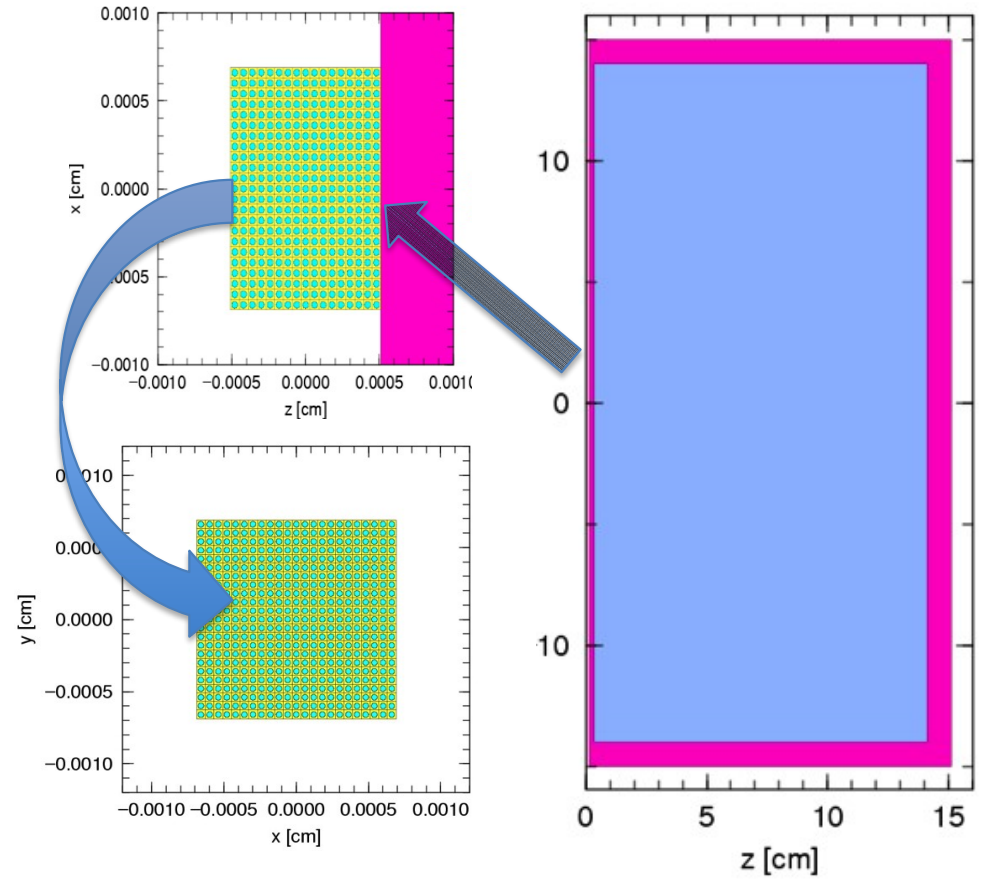

Figure 1. ISO water slab phantom with the film on the front. Right hand side: cross-section of the complete geometry. Top left panel: detail of the cross section of the film on the phantom. Bottom left panel: front view of the film on the phantom.

Figure 1 also shows magnified details in 2D geometry of the microfilm loaded with $\mathrm{CaF}_{2}: \mathrm{Ce}$ spherical grains of $400 \mathrm{~nm}$ diameter The film is shown in the $\mathrm{XZ}$ plane (parallel to the incident radiation beam) and in the XY plane (perpendicular to the incident radiation beam). 
In all figures, white represents air, which is shown in the area around detector and ISO water slab phantom. Magenta represents PMMA, which is shown all around the ISO slab phantom, filled with water shown in Blue-gray. Yellow represents PVC, which is the matrix of the $10.2 \mu \mathrm{m}$-thick microfilm and cyan are the $\mathrm{CaF}_{2}$ :Ce OSL grains.

For the irradiation simulations, we considered a collimated source of Cs-137 monoenergetic gammarays with energy $662 \mathrm{keV}$. A pencil beam with a source radius of $0.001 \mathrm{~cm}$ was used, matching the size of the film. The film was placed perpendicular to the incident beam and the emission of $10^{7}$ monoenergetic gammarays was simulated. Between source and film we simulated the presence of a $100 \mathrm{~cm}$ thick layer of air.

In our simulations, various PHITS tallies were used. The T-Track tally was used to gather information on secondary charged particles, and to check the trajectory, spatial distribution, and flux of charged particles. The T-Deposit tally was used for the deposited energy distribution in the XYZ dimensions in the microfilm and also for the calculations of doses in $\mathrm{MeV} / \mathrm{cm}^{3}$ per source ray.

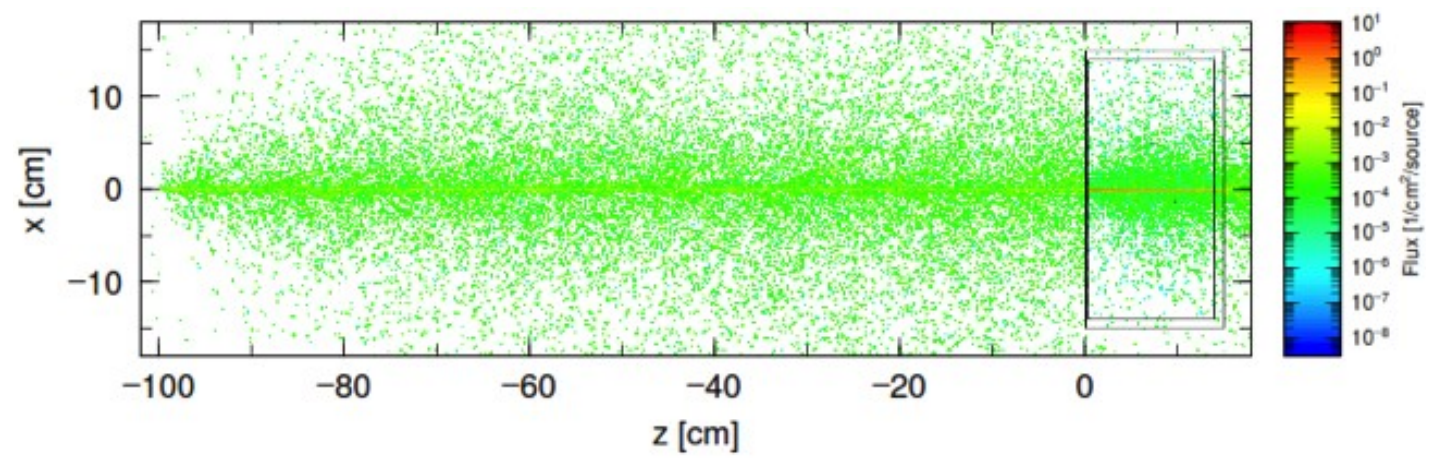

Figure 2. Electron tracks (green) produced by the Cs-137 gamma-rays (red) on their path from the source, through the air, and then across the detectors and the ISO water slab phantom

\section{RESULTS}

The electron tracks produced by the Cs-137 gammarays on their path from the source, through the air, and

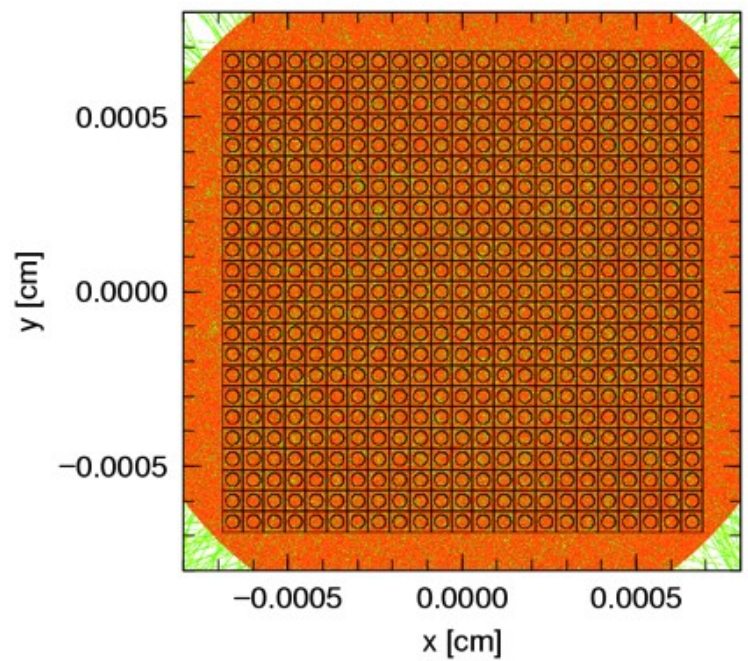

then across the detectors and the ISO water slab phantom is shown in Figure 2. The relative error of all track simulations is smaller than $1 \%$ indicating high precision of the computations.

Figure 3. Front view of the track distributions of primary photon radiation (in red, fluence around $10^{6} \mathrm{~cm}^{-2}$ ) and secondary electrons (in green, fluence around $10^{3} \mathrm{~cm}^{-2}$ )

Cross sections of the track distributions of primary photon radiation (red, fluence around $10^{6} \mathrm{~cm}^{-2}$ ) and of secondary electrons (green, fluence around $10^{3} \mathrm{~cm}^{-2}$ ) in the OSL microfilm detector are shown in Figure 3. The photon beam has a circular shape, hence the rounded edges of the photon distribution in the figure. The electron tracks are due to Compton photon scatter or photoelectric absorption in the microfilm OSL detector and in the ISO phantom. The case shown in Figure 3 refers to a microfilm loaded with $\mathrm{CaF}_{2}: \mathrm{Ce}$ spherical grains with a 400-nm diameter. 
The energy deposition profile across the film is shown in Figure 4, detailing the tracks of electrons from Compton scattering, photoelectric effect, and also
Auger emission produced by the monoenergetic cesium-137 gamma-rays on a microfilm loaded with $400 \mathrm{~nm}$-diameter spherical grains of $\mathrm{CaF}_{2}: \mathrm{Ce}$.

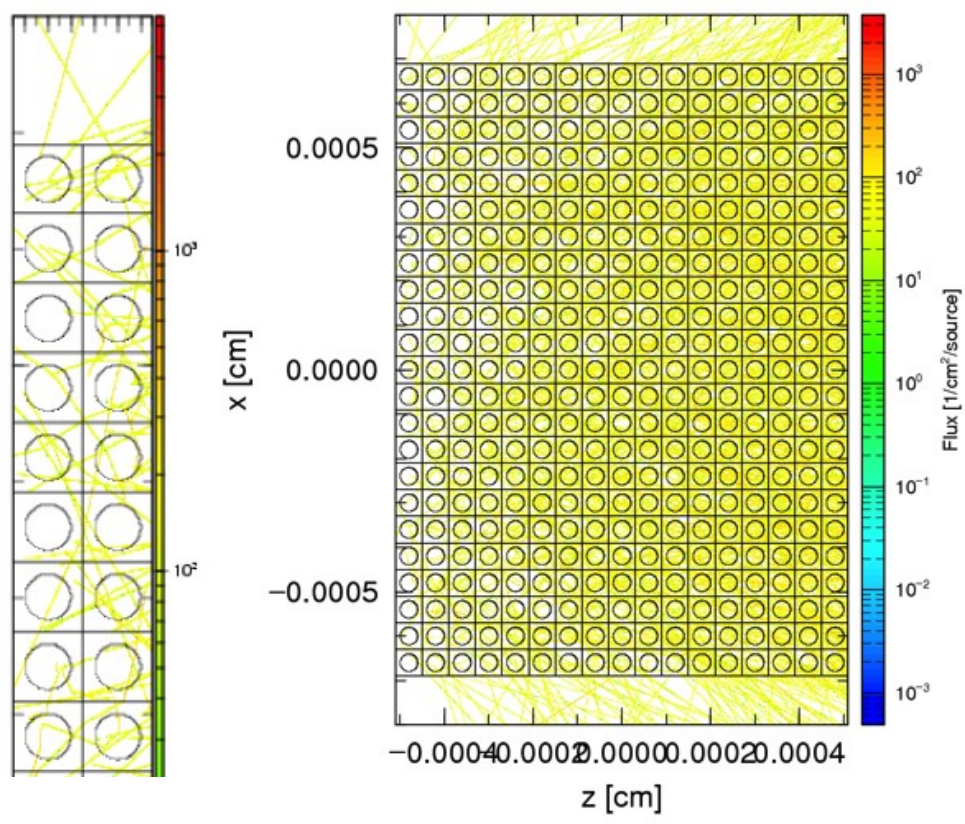

Figure 4. Cross-section of the $10.2 \mu \mathrm{m}$-thick microfilm loaded with $400 \mathrm{~nm}$-diameter spherical grains of $\mathrm{CaF}_{2}$ :Ce showing the electron tracks (around $10^{3} \mathrm{~cm}^{-2}$ ) produced by the Cesium-137 gamma irradiation. The panel on the left hand side shows a magnified view of the first two voxel layers $(1.2 \mu \mathrm{m})$ of the microfilm.

As highlighted by the magnified representation of the two first layers of the film (Figure 4, left hand side), the detector is not in a state of charged-particle equilibrium, but rather a progressive build-up of the dose is clearly visible, i.e., an increasing density of electron tracks as the gamma-ray beam penetrates the microfilm.

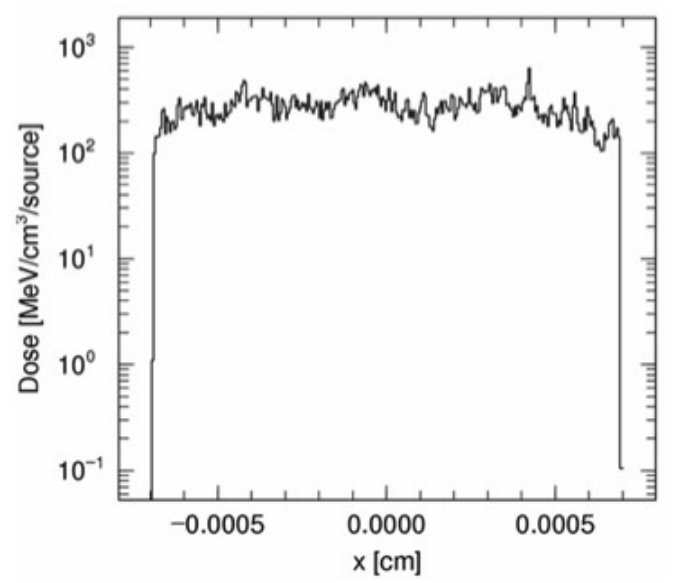

Figure 5. Energy deposition profile in $\mathrm{MeV} / \mathrm{cm}^{3}$ per source gamma ray estimated using a 300 cell reg mesh across the micro-film loaded with $400 \mathrm{~nm}$-diameter spherical grains of $\mathrm{CaF} 2: \mathrm{Ce}$

Figure 5 shows the energy deposition profile in $\mathrm{MeV} / \mathrm{cm}^{3}$ per source gamma ray $\left(1 \mathrm{MeV} / \mathrm{cm}^{3} \sim 10^{-10} \mathrm{~Gy}\right.$, accounting for the effective density of the detector film) estimated using a 300 cell regular mesh across the microfilm loaded with $400 \mathrm{~nm}$-diameter spherical grains of $\mathrm{CaF}_{2}$ : $\mathrm{Ce}$. The figure demonstrates the lack of lateral charged-particle equilibrium in the microfilm.

Table 1. Energy deposition in $\mathrm{MeV} / \mathrm{cm}^{3}$ per source gamma ray in microfilms loaded with $\mathrm{CaF}_{2}$ :Ce grains varying from $50 \mathrm{~nm}$ to $600 \mathrm{~nm}$ and irradiated with a Cs- 137 source

\begin{tabular}{|c|c|c|c|}
\hline $\begin{array}{c}\text { Diameter } \\
\text { of } \mathbf{C a F}_{2}: \mathbf{C} \\
(\mathbf{n m})\end{array}$ & $\begin{array}{c}\text { Deposited } \\
\text { energy } \\
(\mathbf{M e V} / \mathbf{c m} \text { ) }\end{array}$ & $\begin{array}{c}\text { Relative } \\
\text { error \% }\end{array}$ & $\begin{array}{c}\text { Voxel fill } \\
\text { fraction }\end{array}$ \\
\hline 50 & 257.05 & 5.05 & 0.0003 \\
100 & 254.62 & 5.07 & 0.0024 \\
200 & 252.88 & 5.04 & 0.0194 \\
300 & 249.82 & 4.95 & 0.0654 \\
400 & 271.18 & 5.11 & 0.1551 \\
500 & 287.46 & 4.93 & 0.3030 \\
600 & 289.74 & 4.53 & 0.5236 \\
\hline
\end{tabular}

The energy deposition in $\mathrm{MeV} / \mathrm{cm}^{3}$ per source gamma ray in microfilms loaded with $\mathrm{CaF}_{2}$ : $\mathrm{Ce}$ grains varying from $50 \mathrm{~nm}$ to $600 \mathrm{~nm}$ and irradiated with a Cs-137 source is presented in Table 1 . The energy deposition in the OSL film is initially fairly constant with grain size and then increases as the $\mathrm{CaF}_{2}$ :Ce grains get larger to the point of filling $50 \%$ of the voxel volume. It should be noted that the grain size in our actual films is $300 \mathrm{~nm}$ and the volume fill fraction is about 0.02 . 
The statistical uncertainty on the energy deposition was estimated by PHITS to be about $5 \%$ in all cases. This implies that for grain sizes up to almost $400 \mathrm{~nm}$ the impact of the presence of the grains is minimal, i.e., energy deposition values are within statistical uncertainties. In turn, this implies that the dose response of the film is largely determined by the matrix material.

\section{DISCUSSION AND CONCLUSIONS}

The transport and interactions of gamma-rays in a thin film loaded with OSL micro and nanoparticles were studied by Monte Carlo simulations with the Particle and Heavy Ion Transport code System. Our polyvinyl chloride film was treated as an array simulating a small film sample with an area of $13.8 \mu \mathrm{m}$ by $13.8 \mu \mathrm{m}$ and a thickness of $10.2 \mu \mathrm{m}$. The presence of spherical $\mathrm{CaF}_{2}$ : $\mathrm{Ce}$ grains uniformly distributed in the film was simulated. The simulations were performed with different grain diameters, varying from 50 to 600 $\mathrm{nm}$. The film was irradiated in non-equilibrium conditions on the surface of an ISO water slab phantom, and a clear increase in sensitivity was observed with an increase in grain size.

These detectors based on nanoparticles OSL materials dispersed in a polymer matrix are being developed with the aim to achieve a flexible 2D detector offering tissue-equivalence over a broad range of photon energies. In fact, using micro- or nanoparticles offers the advantage that the mean range of secondary electrons generated by photoelectric or Compton interactions within the polymer material is much greater than the OSL particle size, even at low photon energies. The dose is then mainly deposited by secondary electrons generated within the polymer. This allows for the design of tissue-equivalent (TE) dosimeters even with embedded OSL materials, such as $\mathrm{CaF}_{2}$, that exhibit a higher $\mathrm{Z}$-value than tissue. Clearly this is no longer true when approaching a high fill factor. The latter increases the sensitivity of the $\mathrm{CaF}_{2}$ :Ce OSL films at the expense of their tissue equivalence. For this reason, our dosimeter optimization efforts pursue two possible avenues. We can utilize TE luminescent materials in a TE matrix, which is the ultimate solution as it also allows for high fill fractions. Otherwise, we can utilize small fractions of non-TE grains in TE matrices, when high-sensitivity, non-TE materials are available.

Acknowledgement: This research was partly funded by the Brazilian National Research Council $(\mathrm{CNPq})$ under grant number 400430/2013-4. Support is also acknowledged from the Brazilian agencies FAPITEC-SE, CAPES, FAPESP, and from Instituto Nacional de Metrologia das Radiaçoes na Medicina (INCT). S.O. Souza further acknowledges support from $\mathrm{CNPq}$ through grant number 309352/2015-O. F. d'Errico acknowledges support from the Italian Ministry of Education, University and Research under grant 201OSNALEM "Development and application of new materials for ionizing radiation dosimetry”.

\section{REFERENCES}

1. M. Pal et al., "Thermoluminescnce and Optically Stimulated Luminescence Properties of -Irradiated $\mathrm{TiO}_{2}: \mathrm{Yb}$ Nanoparticles," J. Nanosci. Nanotechnol., vol. 9, no. 3, pp. 1851 - 1857, Mar. 2009.

DOI: $10.1166 /$ jnn.2009.369

PMid: 19435049

2. V.S. M. Barros et al., "Thermoluminescent dosimetric properties of $\mathrm{CaF}_{2}: \mathrm{Tm}$ produced by combustion synthesis," J. Radiat. Phys. Chem., vol. 121, pp. 75 - 80, Apr. 2016.

DOI: 10.1016/j.radphyschem.2015.12.017

3. C. A. Perks et al., "Introduction of the. InLight Monitoring Service," Radiat. Prot. Dosim., vol. 125, no. 1-4, pp. 220-223, Mar. 2007.

DOI: $10.1093 / \mathrm{rpd} / \mathrm{ncl} 126$

PMid: 17387125

4. OSL TECHNOLOGY, Landauer, Oxford, UK

Retrieved from:

http://www.landauer.co.uk/whole body osl.html Retrieved on: Dec. 12, 2016

5. S. D. Miller and M. K. Murphy, "Technical performance of the Luxel $\mathrm{Al}_{2} \mathrm{O}_{3}$ : C optically stimulated luminescence dosemeter element at radiation oncology and nuclear accident dose levels," Radiat. Prot. Dosim., vol. 123, no. 4, pp. 435 - 442, Mar. 2007. DOI: $10.1093 / \mathrm{rpd} / \mathrm{ncl} 500$ PMid: 17164274

6. T. E. Burlin, "A general theory of cavity ionization," J. Radiol., vol. 39, no. 466, pp. 727 - 734, Jan. 1966. DOI: $10.1259 / 0007-1285-39-466-727$ PMid: 5927191

7. O. Nakhaei et al., "Synthesis, characterization and study of optical properties of polyvinyl alcohol/ $\mathrm{CaF}_{2}$," Scientia Iranica Transactions, vol. 19, no. 6, pp. 1979 1983, Dec. 2012.

DOI: 10.1016/j.scient.2012.05.008

8. M. Luszik-Bhadra, "Prediction of neutron-induced signals in OSL materials by track structure calculation," Radiat. Meas., vol. 46, no. 12, pp. 1694 - 1697, Dec. 2011. DOI: 10.1016/j.radmeas.2011.03.041

9. S. O. Souza et al., "OSL films for in-vivo entrance dose measurements," Radiat. Meas., press, Jul. 2017. DOI: 10.1016/j.radmeas.2017.07.006

10. B. Azimi et al., "Application of the dry-spinning method to produce poly( $\varepsilon$-caprolactone) fibers containing bovine serum albumin laden gelatin nanoparticles," J. Appl. Polymer Sci. vol. 133, no. 48, pp. $143-148$, Dec. 2016.

DOI: 10.1002/APP.44233

11. H. Iwase et al., "Development of general-purpose particle and heavy ion transport Monte Carlo code," J. Nucl. Sci. Technol., vol. 39, no. 11, pp. $1142-1151$, 2002.

DOI: $10.1080 / 18811248.2002 .9715305$

12. K. Niita et al., "PHITS- a particle and heavy ion transport code system”, Radiat. Meas., vol. 41, pp.10801090, 2006 DOI: 10.1016/j.radmeas.2006.07.013

13. Japan Atomic Energy Agency, Tōkai, Japan, PHITS Version 2.64. Retrieved from: https://phits.jaea.go.jp Retrieved on: Dec. 12, 2016

14. K. Niita et al., "High-energy particle transport code NMTC/JAM," J. Nucl. Instrum. Methd. B, vol. 184, no. 3, pp. $406-420$, Nov. 2001 DOI:10.1016/So168-583X(01)00784-4

15. H. Iwase et al., "Development of heavy ion transport Monte Carlo code", J. Nucl. Instrum. Methd. B, vol. 183 , no. 3-4, pp. $374-382$, Oct. 2001. DOI: $10.1007 / 978-3-642-18211-2 \_15$ 